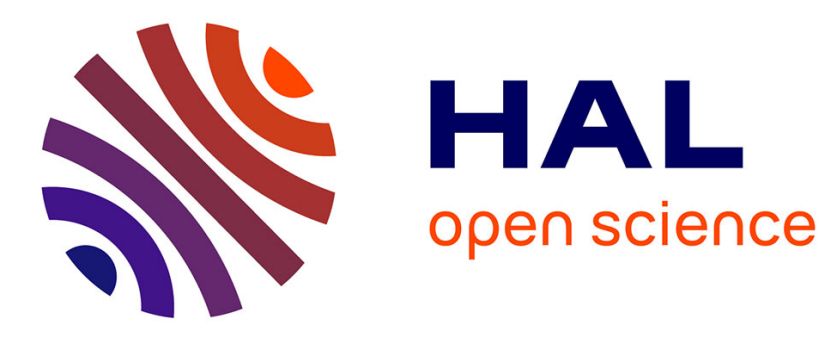

\title{
Efficient Interest Satisfaction in Content Centric Wireless Sensor Networks
}

\author{
Ghada Jaber, Rahim Kacimi, Thierry Gayraud
}

\section{To cite this version:}

Ghada Jaber, Rahim Kacimi, Thierry Gayraud. Efficient Interest Satisfaction in Content Centric Wireless Sensor Networks. 16th IEEE Consumer Communications and Networking Conference (CCNC 2019), Jan 2019, Las-Vegas, United States. pp.1-5, 10.1109/CCNC.2019.8651843 . hal-03012580

\section{HAL Id: hal-03012580 \\ https://hal.science/hal-03012580}

Submitted on 23 Nov 2020

HAL is a multi-disciplinary open access archive for the deposit and dissemination of scientific research documents, whether they are published or not. The documents may come from teaching and research institutions in France or abroad, or from public or private research centers.
L'archive ouverte pluridisciplinaire HAL, est destinée au dépôt et à la diffusion de documents scientifiques de niveau recherche, publiés ou non, émanant des établissements d'enseignement et de recherche français ou étrangers, des laboratoires publics ou privés. 


\title{
Efficient Interest Satisfaction in Content Centric Wireless Sensor Networks
}

\author{
Ghada Jaber ${ }^{1}$, Rahim Kacimi ${ }^{1}$, Thierry Gayraud ${ }^{2}$ \\ IRIT, Université de Toulouse, CNRS, INPT, UPS, UT1, UT2J, France \\ ${ }^{1}$ \{gjaber, kacimi\}@irit.fr \\ 2 gayraud@laas.fr
}

\begin{abstract}
The Content Centric Networking (CCN) is an emerging paradigm that grounds networking primitives on content names rather than node locators. CCN targets seamless mobility, native muticast/multipath support, and content oriented security to better reflect the needs of today users. CCN could greatly improve the efficiency of content delivery also in Wireless Sensor Networks (WSNs). In such a context, we place our attention on the energy efficiency of caching content placement. Our objective is to save energy while achieving a high interest satisfaction rate by study the impact of certain parameters on caching strategy. To this end, we come up with a collaborative on path caching strategy that exploits the node degree and its distance from the source. Through extensive simulations, we demonstrate that our approach achieves a energy efficiency compared to a LCE (Leave Copy Everywhere) while ensuring a good cahe hit then an important interest satosfaction rate.

Index Terms-Wireless sensor networks, informationcentric networking, content-centric networking, content caching, energy efficiency, duty-cycling, interest satisfaction rate.
\end{abstract}

\section{INTRODUCTION}

The massive number of heterogeneous devices made the retrieval of the content by address a hard task and motivated the development of data-centric approaches to networking. Nowadays, people use their devices to share their own contents and they are interested in getting the requested content without having an idea about its location [11]. To face this new demand, the InformationCentric Networking (ICN) paradigm emerged [15]. Many different ICN architectures have been designed so far, all grounded on the common rationale that networking primitives should be based on content names rather than node locators. WSNs represent a very relevant example of ICN system. They are made of wireless devices equipped with sensors that are able to monitor various interests within a target environment with a distributed approach [2]. Whenever, a user is interested by querying the WSN to receive an update of some measurements, he does not care about the node that will ultimately reply to his query but to the actual information that will be returned by the sensor network. In other words, this means that WSNs are ideal candidate to experiment the benefits brought by ICN architectures, as proved by the recent literature [2].
One of the major issues in WSNs is energy consumption because the nodes are usually battery powered. In ICN, distributed in-network caching can alleviate energy consumption due the presence of content copies within the WSN. Consequently, building ICN enabled-WSNs may also help in optimizing energy efficiency [12]. In our work, we explore the node degree and its distance from the source in order to optimize content placement. To formalize our approach, we focus on CCN but we argue that our approach can be applied (with some modifications) to any ICN architecture that works as a network of caches in pull mode. In CCN, a user sends an interest to ask for a specific content and the node with the corresponding content object replies to provide the asked data. Each node maintains three tables: the CS to cache contents, the FIB to store forwarded interests and the PIT to record unsatisfied interests.

In this paper, our objective is to investigate the minimum energy consumption that $\mathrm{CCN}$ achieves while ensuring a high interest satisfaction rate (fraction between the satisfied interests and generated interests in the network). Thus, we come up with acollaborative Caching Strategy Degree and Distance aware (CSDD). To evaluate the energy consumption, we use the mathematical model for energy in CCN/WSNs that we proposed in [9]. Finally, we implement the proposed mechanism and through extensive simulations, we show that our mechanism enhances the energy efficiency compared to LCE and LCD.

The remainder of this paper is organized as follows: in the next section, we present the related work. Section III describes the content caching strategies in CCN. The proposed caching strategy is presented in section IV. Section V details the performance evaluation. Section VII summarizes and concludes the paper.

\section{Related WORKS}

Several contributions on caching in content-centric networking exists. In this paper, we present some as follows:

In [10], authors used the cache capacity as a parameter to select appropriate nodes. In interest packet extra field is used i.e Cache Capacity Value (CCV). When a node receives an Interest and content is not found in its CS, it marks its remaining cache capacity in CCV field and forward Interest. If its CCV is less then it forwards the 
interest and if its CCV is more then it updates CCV in interest and then forward it. When the interest reaches the node that have the content, it assigns the node having the highest CCV as designated node to cache the content.

In [4], the centrality of the node is used as a parameter to select appropriate node. Centrality is measure of importance of a node in communication model. More number of times a node will come on content delivery path more centrality of the node will be. BetwCent chooses a node with the highest betweenness centrality along the content delivery path to store contents.

Bo et al., [6] proposed a collaborative caching strategy for information-centric wireless sensor network (ICNWSN). They proposed a strategy that consists of three parts: the node betweenness based cache size adjustment, the data replacement frequency based cache decision, and the content value based cache replacement algorithm.

To summarize, too many works investigated the problem of caching in CCN. Several works treated this in ICNWSNs. However, to the best of our knowledge, no one proposed a caching strategy that combine at the same time the degree of a node and its position and aim to reduce energy consumption, traveled path and increase cache diversity. In this paper, we aim to combine these parameters in our strategy.

\section{iII. Content Caching in Content-Centric NETWORKING}

\section{A. Caching strategies}

Optimal cache placement is strictly dependent on content placement, request routing, network topology, content replacement, interest arrival rate and caching size [8]. For the proactive content placement, several studies exists. However, the reactive content placement which is our case is more complex due to the behavior of every node when receiving a content from another node.

1) Existing Cache placement policies: In-network caching can present some decision challenges: the cache placement (where to cache), content replacement (which content is to evict from the cache) and request routing (how to redirect requests to optimal cache). In this paper, we treat the cache placement and replacement.

a) Leave Copy Everywhere: Cache management in LCE [14] is defined by its operation of caching data in every node crossed. Part of the practice of the CCN is the ability to make information readily and easily accessible. As a user sends out a request using LCE, the nature of the network serves the interest using hierarchical search and ordering of nodes to acquire a cache-hit.

b) Leave Copy Down: The Leave Copy Down (LCD) [14] is a cache management strategy similar to the drop at the first neighbor process. However, it requires minimal coordination among caching nodes as they can signal to other nodes downstream whether to cache the content or not by simply appending a flag to the delivered content [13].
2) Existing cache replacement policies: The design of cache replacement algorithms is realized for content distribution purposes. When the network becomes stable and the router cache is full, a replacement policy is applied to make room for a new arrived content.

a) Least Frequently Used (LFU): the probability of each item being requested is stationary over time and independent of previous requests. In this case, the optimal replacement policy is the least frequently used, which statically places in cache the $C$ most frequently requested items [13].

b) Least Recently Used (LRU): LRU policy keeps the data recently used and replaces with the other data. LRU has two advantages that makes it very popular, it is very responsive to non-stationary trends, since its replacement decisions are exclusively based on recency and it cannot perform significantly worse than LFU because the ratio between optimal cache hit ratio and LRU cache hit ratio is bounded [13].

c) Random replacement ( $R R$ ): $\mathrm{RR}$ simply replaces a randomly selected item.

\section{On Path Caching Strategy for CCN enabled Wireless Sensor Network}

We consider a wireless sensor network consisting of sensor nodes that interact with the environment and sense the physical data. A sensor nodes that senses and holds the original copy of a data item is called source for that particular data item. Sensor nodes frequently access the data, and cache some data locally to reduce network traffic and data access delay.

\section{A. Cache Admission Control}

When a sensor node receives the requested data or a data item passes through it, a cache admission control is triggered to decide whether it should be stored into the cache of the node or not [5]. Inserting a data item into cache might not always be favorable because incorrect decision can lower the probability of cache hits and also makes poor utilization of the limited storage.

In our strategy, the cache admission decision at a node is based on two conditions: (i) the percentage of path from the source is it greater than $\Delta$ ? (ii) if yes, the nodes does it has a number of neighbors greater than $x$ ?

Therefore our strategy is a 'collaborative Caching Strategy Distance and node Degree aware in content-centric enabled wireless sensor networks' called CSDD.

A trade-off exists between query latency and content accessibility. With a small $\Delta$, the number of copies for each content is high and access delay for this content object is low. On the other hand, with a larger $\Delta$, each content has a small number of copies in intermediate nodes, and the access delay can be longer [5]. However, this depends on the position of the user.

Fig. 1 explains the first condition of our caching strategy and $\Delta$ variation; from how much $\Delta$ from the source, our 


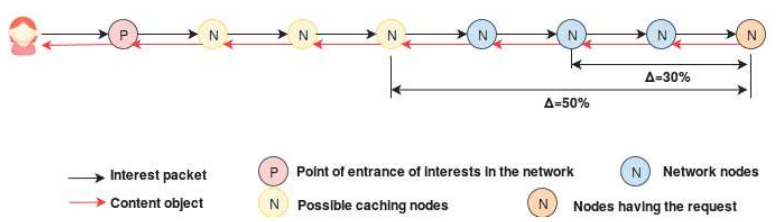

Fig. 1: Caching condition.

strategy decides to cache? In the next section, we will see the impact of this parameter on our caching strategy.

\section{B. Cache replacement policy}

Once the CS is full, our replacement policy relies on replacing the less popular content in the node content store with the new content in the content store. It aims to keep popular contents in the CS. Then our replacement policy is Popularity-based. In addition to that, the interests follow the Zipf distribution.

In our replacement policy, we suppose that popularity denoted by $P$ of content $N 1$ is bigger than the popularity of $N 2$. Then, $P(N 1)>P(N 2)>P(N 3)>\ldots>P(N n)$. Since the cache size is limited, the node only caches the most popular content and evicts the less popular. If two contents of the same popularity exist when applying replacement, our strategy evicts the content having the smallest index for example if $N 8$ and $N 7$ have the same popularity $N 7$ is evicted.

\section{Performance Analysis}

In this section, we examine the performance of CSDD under different degree values $x$ and distance $\Delta$. We then compare it to LCE and LCD, under two replacement policies FIFO and Popularity-based. Moreover, we varied the Zipf exponent $\alpha$.

\section{A. Simulation Set-up}

To implement our scheme, we use CCNx_Contiki framework [12] and we extend it to follow the requirements of our mechanism. Contiki [7] is an open source operating system for the Internet of Things with memoryconstrained embedded systems and WSNs. Simulations are conducted using Cooja simulator. Fig. 2 shows how our

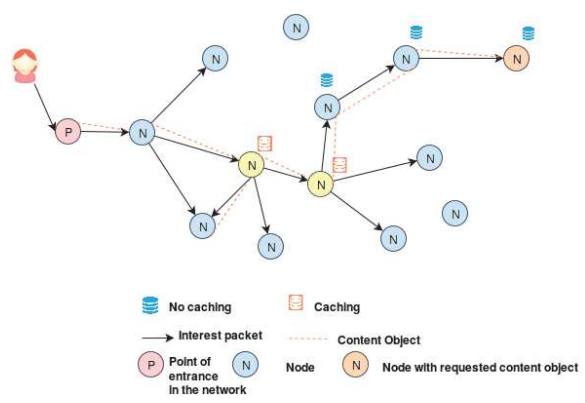

Fig. 2: Caching strategy.

strategy works if a user send an interest for a content and if $\Delta$ is equal to $50 \%$ and $x>=3$. When the requested content object is found, it is sent back to the user. Meanwhile, it is cached in nodes that fulfill the two condition related to the degree $x$ and position from the source $\Delta$.

\begin{tabular}{lc}
\hline Simulation Parameters & Value \\
\hline Area & $500 \mathrm{~m} \times 500 \mathrm{~m}$ \\
Simulation duration & $3600 \mathrm{~s}$ \\
Radio coverage range & $100 \mathrm{~m}$ \\
Initial energy & $2 \mathrm{~J}$ \\
Cache size & $6(30 \%)$ \\
Number of generated types of content & 20 \\
Number of entrance points in the network & 4 \\
Values of $\alpha$ & $0.2,0.6,1,1.4,1.8$ \\
\hline
\end{tabular}

TABLE I: Simulation parameters

Let consider a WSN deployed on an area of $100 \times 100 \mathrm{~m}$. The number of nodes varies from 80 to 240. All the sensor nodes are static and have the same detection radius. In addition, all the communication links are bidirectional. Different types of content are generated by the network. Users are modeled by application agents which polls interests. Simulation parameters are detailed in Table I. For the sake of illustration, we take into account not only the energy consumption modeled in the previous section but also the energy consumed due to the interests forwarding. To evaluate the energy consumption, we adopt the example of power consumption of Tmote sky.

\section{B. Evaluation metrics}

We use four main performance metrics for evaluation:

- Energy consumption: the total energy consumed by the network [9].

$$
\begin{aligned}
& \bar{E}_{i}^{n}=\sum_{i=1}^{K}\left(p_{i}\left(1-e^{-p_{i} \tau_{i}}\right)\left(\alpha t_{t x_{i}} P_{t x}+E_{p_{i}}^{n}\right)\right.
\end{aligned}
$$

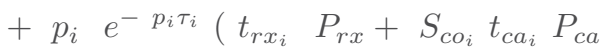

$$
\begin{aligned}
& \left.\left.+t_{t x_{i}} P_{t x}+E_{p_{i}}^{n}\right)+\left(1-p_{i}\right) E_{p_{i}}^{n}\right)
\end{aligned}
$$

- Cache hit: measured on a path when looking for a content [3] which reflects the interest satisfaction rate.

$$
\text { Cache hit }=\frac{\sum_{i=1}^{N} \text { hits }_{i}}{\sum_{i=1}^{N} \text { hits }_{i}+\sum_{i=1}^{N} \text { miss }_{i}}
$$

\section{Simulation Results}

In this study, we implement two cache replacement policies: the FIFO and the Popularity-based strategies. Since our interests follow Zipf distribution, we consider that different $\alpha$ describes different scenarios. 
Energy consumption: Figure 3 plots the results of energy consumption for the different strategies under different variation of $\alpha$ for two replacement policies: FIFO and Popularity-based.

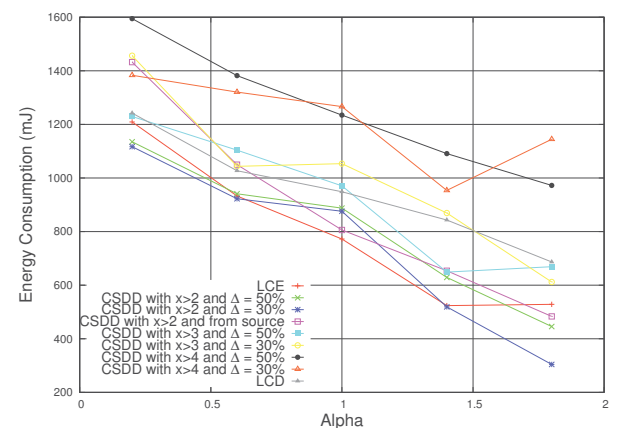

(a) Energy consumption when using FIFO.

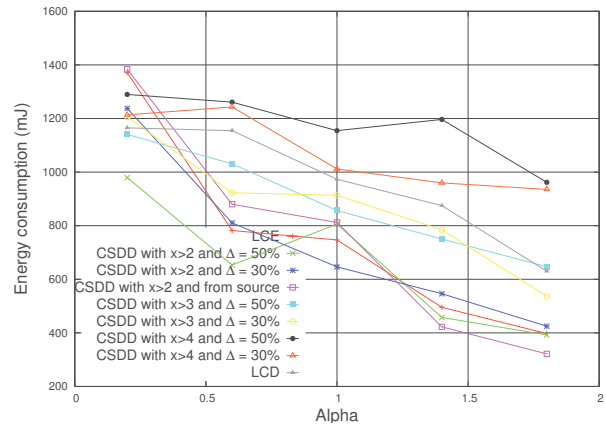

(b) Energy consumption when using Popularitybased.

Fig. 3: Energy consumption for all the strategies when using FIFO and Popularity-based replacement policies for 80 nodes.

For both replacement policies, it is noticed that when $\alpha$ increases the energy consumption decreases for all the strategies under both replacement policies. Indeed, when $\alpha$ increases, popular contents will be more requested by users and they will be cached more in intermediate nodes. Consequently, most of the requests cross a shorter path. In low popularity scenarios, all the strategies consumes more energy. This is due to the fact that the contents are almost requested with the same rate. Then, the replacement happens frequently increasing energy consumption.

It is also worth to note that under the FIFO replacement policy, all the strategies consume more energy. This is explained by the fact that since the interests follow Zipf distribution in both cases, when using FIFO, the probability of replacement is the same for all the contents. Nevertheless, when using the Popularity-based policy, popular contents will be kept in the caches. Therefore, the network consumes less energy consumption since requested contents will be in the cache for longer time. For instance, for $\alpha=0.4$, under FIFO, LCE consumes about $1230 \mathrm{~mJ}$ and $\operatorname{CSDD}(x>2$ and $\Delta=30 \%)$ consumes slightly more than $950 \mathrm{~mJ}$. Yet, under
Popularity-based policy, CSDD $(x>2$ and $\Delta=30 \%)$ dissipates just $890 \mathrm{~mJ}$ and LCE consumes $1050 \mathrm{~mJ}$.

For low popularities, CSDD with a node degree higher than 2 and a caching distance from $30 \%$ or $50 \%$ consumes less energy than LCE, LCD and CSDD with a degree of 3 or 4 . Since nodes with a degree higher than 3 represents only $16 \%$ of the network nodes, contents will not be cached on a lot of nodes then, requests will be forwarded to many nodes to find the content. Then, CSDD with a degree of 3 or 4 consumes more energy.

Cache hit ratio: We also studied the cache hit ratio for all the strategies under different popularity distribution when using both FIFO and Popularity-based replacement policies.

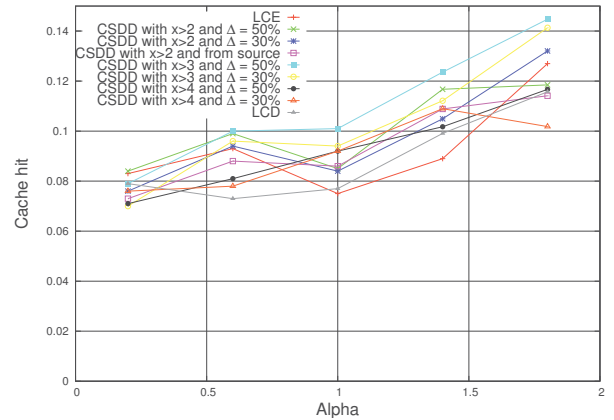

(a) Cache hit when using FIFO

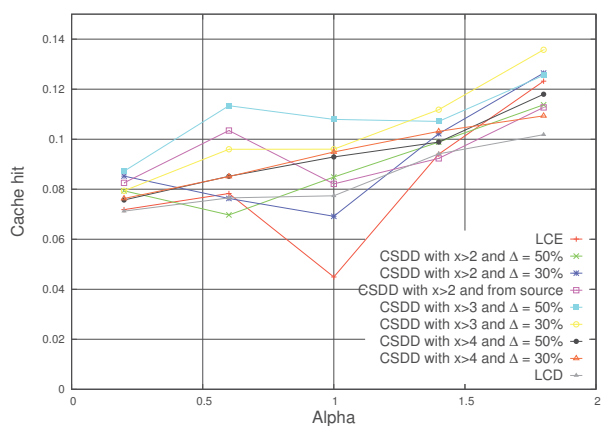

(b) Cache hit when using Popularity-based.

Fig. 4: Cache hit for all the strategies when using FIFO and Popularity-based replacement policies.

The results plotted in Fig 4 have shown that when the popularity increases, the cache hit increases when using FIFO and Popularity-based. Indeed, when the $\alpha$ increases, the popularity of certain contents increases and they will be more requested hence they will be more available on network node.

In Fig. 4a, for $\alpha=0.2$ when FIFO is used, we observe that LCE outperforms almost all the strategies (except CSDD with $x>2$ and a $\Delta=30 \%$ or $\Delta=50 \%$ ). This is due to the uniform distribution of interest and to the maximum caching in nodes. However, in high popularity, CSDD with $x>3$ and a $\Delta=30 \%$ or $\Delta=50 \%$ reports better performance than LCE. 
For Popularity-based replacement policy, for low popularity, this time LCE presents the worst result because it is caching everywhere the same contents. However, in high popularity, it is achieving the better results since during the rush hours, there are a lot of interests sent on the network requesting the most popular contents. So, it is better to make them available. However, LCE still does not outperform CSDD with an $x>2$ or $x>3$ in this case. Therefore, we notice that the values of the caching are low since, in a wireless network, the interests are widely diffused increasing the number of miss since the requested content is not cached in all the network node. Then, they will be recording cache miss and decreasing the cache hit ratio. Increasing cache hit will generate the increasing in the interest satisfaction rate.

Finally, we argue that the choice of the degree must be coherent with the percentage of nodes having this degree. For instance, the results showed that the degree $x>4$ realized bad results since it just represents $4 \%$ of the network nodes. Then, the number of potential candidates on which caching is realized, is low.

\section{CONCLUSion}

In this paper, we proposed a new caching strategy that aims to find an optimal way to cache the content in order to realize better network performance. For this, we started by presenting different existing caching strategy in the literature that we implemented further. After that, we proposed CSDD an on-path caching strategy in contentcentric enabled wireless sensor networks with two parameters to vary (node degree and its distance from the source node). Finally, we presented the simulation results in order to show the impact of CSDD on energy consumption and cache hit. An interesting direction for future work is to study the performance of our strategy in terms of replacement rate, cache diversity, and network lifetime. Besides, from an experimental view, we will implement our mechanism on a the real platform of neOCampus network.

\section{ACKNOWLEDGMENT}

This work is supported jointly by the neOCampus research grant [1] and the Occitanie Province.

\section{REFERENCES}

[1] NeocampusLabs. https://www.irit.fr/neocampus/fr/. Online; accessed 22 Novembre 2017.

[2] Marica Amadeo, Claudia Campo, Antonella Molinaro, and Nathalie Mitton. Named Data Networking: a Natural Design for Data Collection in Wireless Sensor Networks. In IEEE Wireless Days (WD), Valencia, Spain, November 2013.

[3] César Bernardini, Thomas Silverston, and Olivier Festor. A comparison of caching strategies for content centric networking. In Global Communications
Conference (GLOBECOM), 2015 IEEE, pages 1-6. IEEE, 2015.

[4] Wei Koong Chai, Diliang He, Ioannis Psaras, and George Pavlou. Cache less for more in informationcentric networks (extended version). Computer Communications, 36(7):758-770, 2013.

[5] Narottam Chand. Cooperative data caching in wsn. World Academy of Science, Engineering and Technology, 63:90-94, 2012.

[6] Bo Chen, Liang Liu, Zhao Zhang, Wenbo Yang, and Huadong Ma. Brr-cvr: A collaborative caching strategy for information-centric wireless sensor networks. In Mobile Ad-Hoc and Sensor Networks (MSN), 2016 12th International Conference on, pages 31-38. IEEE, 2016.

[7] Shuai Gao, Hongke Zhang, and Beichuan Zhang. Energy efficient interest forwarding in ndn-based wireless sensor networks. Mobile Information Systems, 2016, 2016.

[8] Hashim A. Hashim, B.O. Ayinde, and M.A. Abido. Optimal placement of relay nodes in wireless sensor network using artificial bee colony algorithm. J. Netw. Comput. Appl., 64(C):239-248, April 2016.

[9] Ghada Jaber, Rahim Kacimi, Luigi Alfredo Grieco, and Thierry Gayraud. An adaptive duty-cycle mechanism for energy efficient wireless sensor networks, based on information centric networking design. Wireless Networks, Sep 2018.

[10] Dabin Kim, Sung-Won Lee, Young-Bae Ko, and JaeHoon Kim. Cache capacity-aware content centric networking under flash crowds. J. Netw. Comput. Appl., 50(C):101-113, April 2015.

[11] Jihoon Lee and Daeyoub Kim. Proxy-based mobility management scheme in mobile content centric networking (ccn) environments. IEEE Transactions on Consumer Electronics, 57(2), 2011.

[12] Bilel Saadallah, Abdelkader Lahmadi, and Olivier Festor. CCNx for Contiki: implementation details. PhD thesis, INRIA, 2012.

[13] Lorenzo Saino. On the Design of Efficient Caching Systems. $\mathrm{PhD}$ thesis, University College London, 2015.

[14] A. Suki, M. ArifSuhaidi, and HassanSuhaidi HassanIbrahim AbdullahiIbrahim Abdullahi. Cache replacement positions in information-centric network. In International Conference on Internet Applications, Protocol and Services (NETAPPS2015), Oct. 2015.

[15] George Xylomenos, Christopher N Ververidis, Vasilios A Siris, Nikos Fotiou, Christos Tsilopoulos, Xenofon Vasilakos, Konstantinos V Katsaros, and George C Polyzos. A survey of information-centric networking research. IEEE Communications Surveys $\&$ Tutorials, 16(2):1024-1049. 